\title{
$\pi-\Xi$ correlations in Au-Au collisions at STAR
}

Petr Chaloupka ${ }^{a}$ (for the STAR collaboration)

${ }^{a}$ Nuclear Physics Institute, Academy of Sciences of the Czech Republic, 25068 Rez near Prague, Czech Republic

We present $\pi-\Xi$ correlation analysis in Au-Au collisions at $\sqrt{s_{N N}}=200 \mathrm{GeV}$ and $\sqrt{s_{N N}}=62.4 \mathrm{GeV}$, performed using the STAR detector at RHIC. A $\Xi^{*}(1530)$ resonance signal is observed for the first time in Au-Au collisions. Experimental data are compared with theoretical predictions. The strength of the $\Xi^{*}$ peak is reproduced in the correlation function assuming that $\pi$ and $\Xi$ emerge from a system in collective expansion.

\section{Introduction}

Heavy-ion collisions allow the study of strongly interacting matter in extreme conditions, similar to those of early universe. At the energy densities achieved in Au-Au collisions at RHIC[ 1 the produced matter is expected to be governed by partonic degrees of freedom.

Current data on spectra and elliptic flow from Au-Au collisions at $\sqrt{s_{N N}}=200 \mathrm{GeV}$ [ 2, 3, 4] and $\sqrt{s_{N N}}=130 \mathrm{GeV}$ [ [5, 6] suggest that the hot and dense system created in the collision builds up substantial collective behavior leading to a rapid transverse expansion. The properties of the induced flow will differ depending on whether the collectivity was achieved on a partonic or hadronic level. Elliptic flow and spectra data of $\Xi[7$, 8] suggest that $\Xi$ s pick up less flow than other hadrons $(\bar{\Lambda}, K, p)$. Because $\Xi$ hadronic cross-section is presumably small, $\Xi$ s are expected to undergo few interactions in the hadronic phase, hence picking up less flow and decoupling earlier than $\bar{\Lambda}, K, p$. Therefore $\Xi$ s are likely to carry more direct information about the partonic stage than other hadrons.

Measurements of final state particle correlations may yield important insight into the properties of matter created in the earlier collision times and evolution of the system. Nonidentical particle correlation analyses were suggested [9] to provide a way of measuring space-time properties of the particle-emitting source. Compared to standard identical particle interferometry (sometimes called HBT), which measures one particle species source size, the non-identical particle correlation function provides a way to measure relative space-time emission asymmetry among two particles.

Further insight into the dynamics is provided when studying the influence of flow on the space-time freeze-out distribution of the source using a correlation function. Since flow induces a strong correlation between particle velocities and emission points, heavier particles are expected to be emitted on average closer to the surface of the source than the light ones. This leads to an effective decrease of measured HBT radii and shifts of average emission points of different particle species relative to each other [10]. The study of two particle systems with a large mass difference, like $\pi-\Xi$, is therefore of high interest 
when studying expansion dynamics. For a detailed discussion of flow and its effect on particle correlation measurements see [4, 10].

\section{Data}

STAR main detector, the Time Projection Chamber, detects charged particles emerging from primary as well as secondary vertices. Pions, kaons and protons are identified via their specific energy loss $(\mathrm{dE} / \mathrm{dx})$. We topologically reconstruct charged $\Xi$ hyperons, which decay via $\Xi \rightarrow \Lambda+\pi$, and subsequently $\Lambda \rightarrow \pi+p$, into proton and two pions.

The $\pi-\Xi$ correlation function $C\left(k^{*}\right)$ was analyzed in the pair rest frame, where $\vec{k}^{*}=\vec{p}_{\pi}^{*}=-\vec{p}_{\Xi}^{*}$. The distribution of real $\pi-\Xi$ pairs from individual events was divided by a mixed-event pair distribution, in which each particle was taken from a different event. In order to have a well-defined baseline, events for mixing were divided into individual classes in multiplicity and primary vertex position. To avoid fake correlations coming from a mismatched $\Xi$ bachelor pion (pion from $\Xi \rightarrow \Lambda+\pi$ decay) we require the momentum difference between the primary and bachelor pion to be greater than $0.05 \mathrm{GeV} / \mathrm{c}$. For this analysis only primary pions with $|y|<0.5$ were accepted.

Two data sets of Au-Au collisions at energies $\sqrt{s_{N N}}=200 \mathrm{GeV}$ and $\sqrt{s_{N N}}=62.4 \mathrm{GeV}$ were used. Events recorded at $200 \mathrm{GeV}$ consist of two datasets: the minimum bias sample covers centrality $0 \%-80 \%$, while the other set contains $10 \%$ most central collisions. The $62.4 \mathrm{GeV}$ minimum bias dataset consists of events with centrality between $0 \%-80 \%$.

The correlation function was corrected for pair purity, defined as the product of the $\pi$ and $\Xi$ purities. The purity of the $\Xi$ sample, was estimated from the signal to combinatoric background ratio in invariant mass distribution as a function of $p_{t}$. The pion sample purity was estimated from $\lambda$ measured in $\pi-\pi$ HBT analysis [114. The influence of the momentum resolution is still under study and has not been corrected for.

\section{Results}

In Figure 1 we present the preliminary results on $C\left(k^{*}\right)$ for all four combinations of $\pi^{ \pm}-\Xi^{ \pm}$pairs from the $10 \%$ most central $200 \mathrm{GeV}$ Au-Au collisions. In the low $k^{*}$ region $\left(k^{*}<0.05 \mathrm{GeV} / \mathrm{c}\right)$ the correlation function is dominated by the Coulomb interaction. Since $C\left(k^{*}\right)$ is, within statistical errors, the same for $\pi^{+}-\Xi^{-}, \pi^{-}-\Xi^{+}$and $\pi^{+}-\Xi^{+}, \pi^{-}-\Xi^{-}$pairs, correspondingly, the data with like and unlike-sign pairs are combined, in order to improve the statistics.

In Figure 2 the centrality dependence of $C\left(k^{*}\right)$ for unlike-sign pairs at $62.4 \mathrm{GeV}$ and $200 \mathrm{GeV}$ is presented. For statistics reasons only three centrality bins are used. Even though the analysis is statistically challenging, the correlation functions exhibit the same general features at all centrality bins and energies. The Coulomb region is mostly affected by the low statistics which does not make it possible to observe conclusively any centrality dependence. However a peak at $k^{*} \approx 0.15 \mathrm{GeV} / \mathrm{c}$, corresponding to $\Xi^{*}(1530) \rightarrow \Xi+\pi$ decay is clearly visible. $k^{*}$ is directly connected to the invariant parent particle mass $M: k^{*}=\sqrt{\left[M^{2}-\left(m_{1}-m_{2}\right)^{2}\right]\left[M^{2}-\left(m_{1}+m_{2}\right)^{2}\right]} / 2 M$. As a crosscheck we verify that the $\Xi^{*}$ peak is also visible in the invariant mass distribution as shown in Figure 3. The distributions show a visible $\Xi^{*}$ peak in both datasets. No extraction of the yield has been attempted so far. Most importantly, the $\Xi^{*}$ peak in $C\left(k^{*}\right)$ shows significant centrality dependence. 


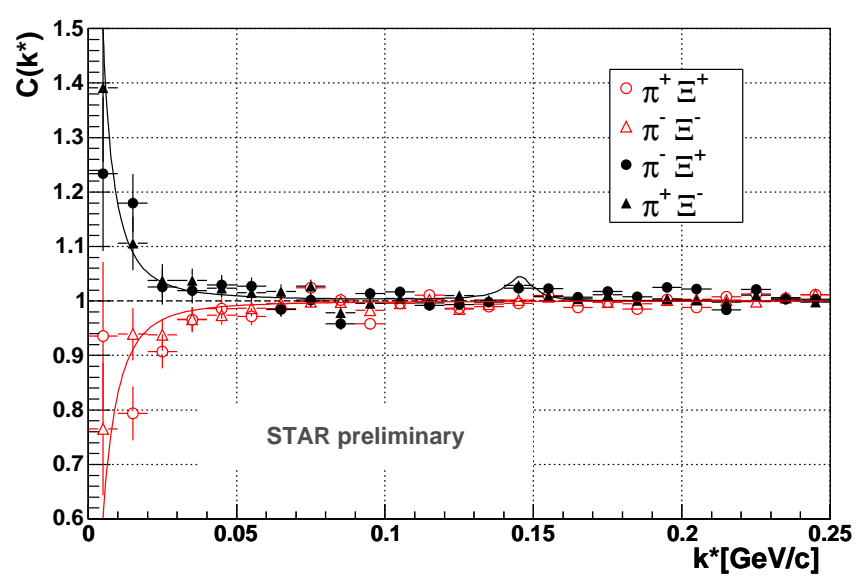

Figure 1. $\pi-\Xi$ correlation function for $10 \%$ most central collisions at $200 \mathrm{GeV}$. Triangles and circles are experimental data, solid lines are theoretical predictions.

\section{Theory comparison}

The correlation function $C\left(\vec{k}^{*}\right)$ of two particles with momenta $\vec{p}_{a}$ and $\vec{p}_{b}$ is determined by source properties, characterized by $g\left(\vec{r}^{*}\right)$, the normalized probability of two particles' emission points being separated by a distance $\vec{r}^{*}$ in the pair's center-of-mass frame. $C\left(\vec{k}^{*}\right)=\int g\left(\vec{r}^{*}\right)\left|\psi\left(\vec{k}^{*}, \vec{r}^{*}\right)\right|^{2} d^{3} r^{*}$, where $\vec{k}^{*}=\vec{p}_{a}^{*}=-\vec{p}_{b}^{*}$ is the particle momentum in the pair rest frame.

Thus knowledge of the wave function $\psi\left(\vec{k}^{*}, \vec{r}^{*}\right)$ of two interacting particles, allows to predict $C\left(\vec{k}^{*}\right)$. A method to evaluate $\psi\left(\vec{k}^{*}, \vec{r}^{*}\right)$ for different systems of non-identical particles (including $\pi-\Xi$ system), incorporating the Coulomb and strong interactions has been recently proposed in [11] (generalizing the method in [12]).

Theoretical predictions for $C\left(\left|\vec{k}^{*}\right|\right)$ are shown in Figure 1 and Figure 2 together with corresponding real data. Standard $R_{\text {out }}, R_{\text {side }}, R_{\text {long }}$ source parameterization[13] has been used for both $\pi$ and $\Xi$ sources. The values were obtained from hydro-inspired blast wave calculations [10], with the assumption that $\Xi$ s flow as pions, kaons and protons. The parameters for blast wave fit were obtained from fitting spectra[ 2] and $\pi-\pi$ HBT radii[ 14 extracted from $200 \mathrm{GeV}$ Au-Au STAR data. The parameters used in he blast wave fit for different centralities can be found in [14].

Similarly to experimental data the theoretical $C\left(k^{*}\right)$ of unlike-sign pairs shows peak corresponding to $\Xi^{*}(1530)$ resonance. Moreover the peak strength exhibits significant sensitivity to the size of the source.

\section{Conclusions}

We have presented first measurements of the $\pi-\Xi$ correlation function $C\left(k^{*}\right)$ in heavy ion collisions. The results, though still preliminary, show clearly the effects of Coulomb and strong interactions among produced $\pi$ and $\Xi$. In particular, strong interactions leading to the $\Xi^{*}(1530)$ resonance signal is observed for the first time in heavy-ion collisions. Moreover, in the region of $\Xi^{*}(1530)$, the $C\left(k^{*}\right)$ shows much stronger sensitivity to the source size than in the Coulomb region.

The theoretical prediction, under assumption of both particles exhibiting transverse radial flow, reproduces experimental data within statistical errors. However, to unam- 


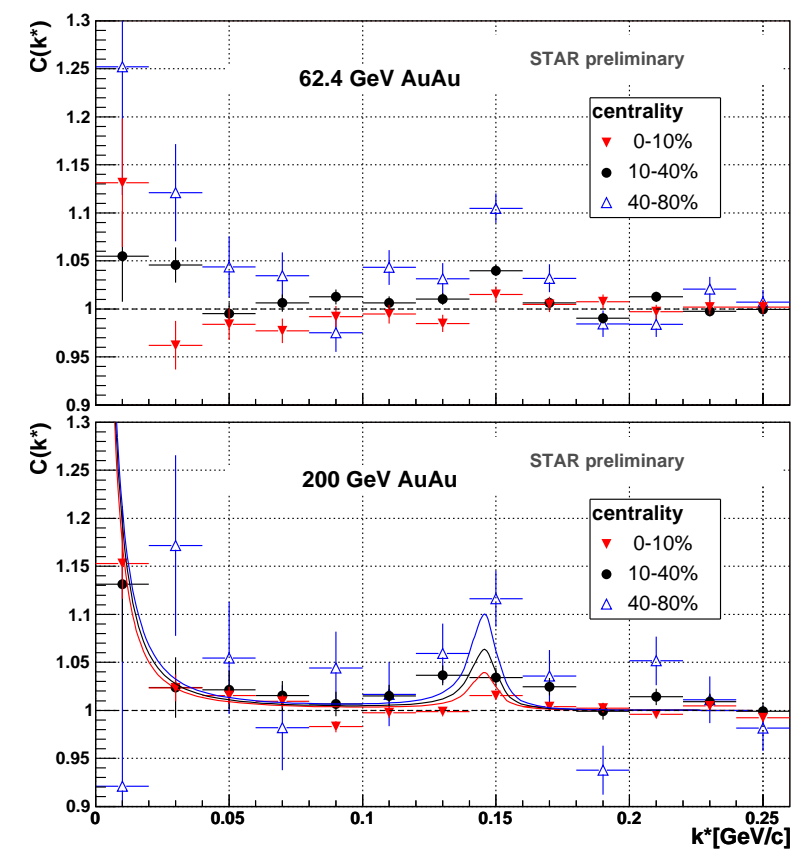

Figure 2. Centrality dependence of $C\left(k^{*}\right)$ for unlike-sign $\pi-\Xi$ pairs; upper panel: at $62.4 \mathrm{GeV}$, lower panel: at $200 \mathrm{GeV}$. Solid lines show theoretical predictions for corresponding centrality bins.

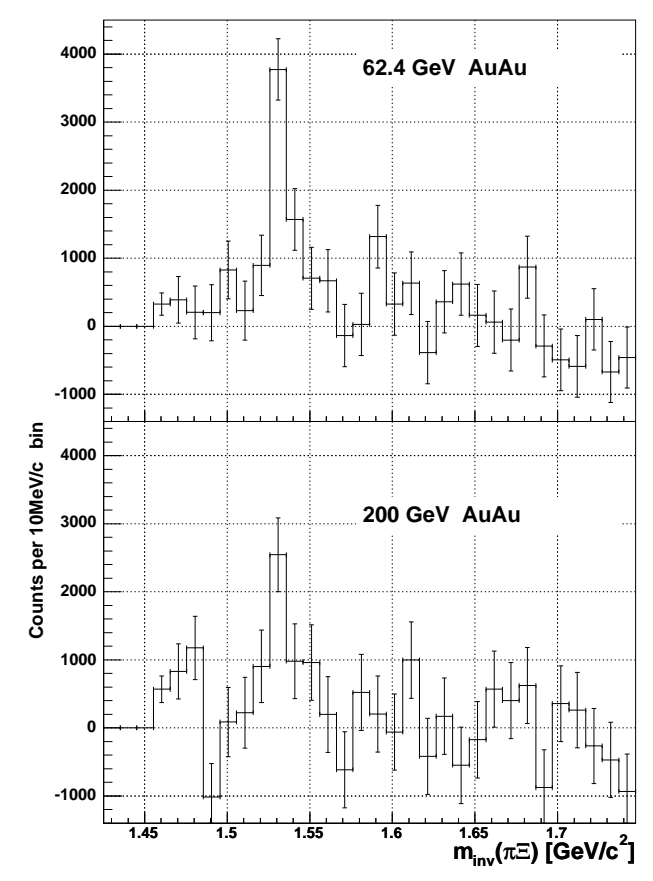

Figure 3. $\pi-\Xi$ invariant mass distribution of unlike-sign pairs; upper panel: at $62.4 \mathrm{GeV}$, lower panel: at $200 \mathrm{GeV}$.

biguously conclude on $\Xi$ flow, it is necessary to probe shifts of average space-time emission points constructing $C_{+}\left(k^{*}\right) / C_{-}\left(k^{*}\right)$ ratio[ 9 . We expect that sufficient statistics to perform this study will be available from RHIC 2004 data run.

\section{Acknowledgements}

The author wishes to thank S. Pratt for making his code of model calculations available to us. This work was supported by the Grant Agency of the Czech Republic grant 202/04/0973.

\section{REFERENCES}

1. Gyulassy M 2004 J. Phys. G: Nucl. Part. Phys. 30 S911-S918

2. Adams J 2004 Phys.Rev.Lett. 92112301

3. Adams J 2004 arXiv:nucl-ex/0409033

4. Retière F 2004 J. Phys. G: Nucl. Part. Phys. 30 S837-S834

5. Adler C 2001 Phys.Rev.Lett. 87112303

6. Adler C 2002 Phys Rev. C 66034904

7. Castillo J 2004 J. Phys. G: Nucl. Part. Phys. 30 S120\%-S1211

8. Estienne $\mathrm{M}$, these proceedings

9. Lednický R, Lyuboshitz V L, Erazmus B, Nouais D 1996Phys Lett B 373 30-34

10. Retière F, Lisa M 2003 arXiv:nucl-ex/0312024

11. Pratt S, Petricioni S 2003 Phys.Rev.C 68054901

12. Lednicky R, Lyuboshitz V.L. 1982 Sov. J. Nucl. Phys. 35770

13. Pratt S, Csörgö T, and Zimanyi J 1990 Phys. Rev. C 42, 2646 (1990); Bertsch G, Gong M, and Tohyama M 1988 Phys. Rev. C 371896

14. Adams J (STAR collaboration) STAR HBT PRC - to be published 